\title{
Calidad de vida relacionada con características sociodemográficas y clínicas en niños con parálisis cerebral
}

\section{Quality of life related to socio-demographic and clinical characteristics in children with cerebral palsy}

\author{
Ana Marcela Bolaños-Roldán ${ }^{1}$ \\ 1. Universidad del Valle. Cali, Colombia. Correo: ana.marcela.bolanos@correounivalle.edu.co - https://orcid.org/0000-0002-9222-8541 \\ Tipología: artículo de investigación científica y tecnológica \\ Para citar este artículo: Bolaños-Roldán AM. Calidad de vida relacionada con características sociodemográficas y clínicas en niños con parálisis cerebral. Duazary. 2020 abril - \\ junio; 17 (2): 20 - 31. Doi: http://dx.doi.org/10.21676/2389783X.3230 \\ Recibido en marzo 27 de 2019 \\ Aceptado en agosto 07 de 2019 \\ Publicado en línea en diciembre 1 de 2019
}

\section{RESUMEN}

Palabras

clave: calidad

de vida;

parálisis

cerebral; niño.

Keywords:

Quality of life; cerebral palsy; child.
La parálisis cerebral (PC) es la causa más frecuente de discapacidad en la infancia. El objetivo de esta investigación fue determinar la calidad de vida relacionada con la salud (CVRS) de niños y niñas entre 8 y 12 años con PC y relacionarla con características sociodemográficas y clínicas. En este estudio descriptivo correlacional se aplicaron dos cuestionarios: uno con información sociodemográfica y clínica, y el KIDSCREEN 27, a una población de 100 niños con PC o a sus cuidadores principales, para el caso de aquellos con dificultades cognitivas o comunicativas. Las dimensiones de entorno escolar y estado de ánimo y sentimientos fueron mejor percibidas. En los niños más pequeños (8 a 9 años) se encontró una mejor percepción sobre la actividad física y la vida familiar y el tiempo libre, mientras que los niños mayores (10 a 12 años) se refirieron al dominio de apoyo social y amigos. Los niños de estrato socioeconómico alto dieron mayor puntuación en todos los dominios de calidad de vida, al igual que los niños menos comprometidos en su función motora gruesa. El más alto nivel educativo de los cuidadores, el estrato socioeconómico alto y una menor severidad en el compromiso motor en los niños con PC se relacionan con una mejor calidad de vida.

\section{ABSTRACT}

Cerebral Palsy (CP) is the most frequent cause of disability that appears in the in early childhood. The aim was to describe health-related quality of life (QoL) of boys and girls between 8 and 12 years old with $\mathrm{CP}$ and its relation to demographic and clinical characteristics. This is a descriptive correlational study using a questionnaire on demographic factors and clinical characteristics and the Kidscreen-27. A group of 100 children with CP or their main caregivers, (for those who had cognitive or communication difficulties). In this group the best perceived dimensions of QoL were school environment, mood state and feelings. The younger children ( 8 to 9 years old) reported better perception of physical activity, family life, and free time. Older children (10 to 12 years old) had better scores in the social support and friendship dimension. Children from higher socioeconomic strata presented better scores in all domains of QoL. Children with better gross motor function displayed better scores in all domains of QoL. In this study children with better QoL had caregivers with better education level, better socioeconomic strata and they had better gross motor function. 


\section{INTRODUCCIÓN}

La parálisis cerebral (PC) es la causa más frecuente de discapacidad motora en la infancia ${ }^{1}$. La PC se describe como un grupo de trastornos de la postura y el movimiento causados por una lesión en el cerebro inmaduro; no es progresiva y puede estar asociada a problemas sensoriales, perceptuales, cognitivos, comunicativos y de la conducta ${ }^{2}$. Se considera que la incidencia es mayor en lactantes que fueron moderadamente prematuros en comparación con los recién nacidos a término, encontrándose una disminución en la incidencia con el incremento de la edad gestacional ${ }^{3}$. La prevalencia global de PC oscila entre 1,5 a 2,5 por cada 1.000 nacidos vivos y es notoriamente disímil entre países desarrollados y aquellos en condiciones de pobreza: se refiere que en África es de hasta 10 casos por cada 1.000 nacidos vivos ${ }^{4}$, en tanto que en países en desarrollo se observan hasta 5 casos por 1.000 nacidos vivos $^{5}$ (en el Perú, por ejemplo, existe una prevalencia de 5,2 niños con PC por cada 1.000 nacidos vivos ${ }^{6}$ ). En la Línea Base del Observatorio Nacional De Discapacidad de Colombia se informó que para el año 2012, en los grupos de edad de 0 a 17 años, el 1,24\% de esta población tenía algún tipo de discapacidad; sin embargo, no se encontró información específica sobre la prevalencia de la $\mathrm{PC}^{7}$.

La discapacidad generada por la PC puede ser apenas apreciable o manifestarse de manera severa, lo que puede impactar en la calidad de vida (CV) de los niños y sus familias. El concepto de $\mathrm{CV}$, según la OMS, se refiere a la percepción que cada individuo tiene de su posición en la vida en el contexto cultural, así como del sistema de valores en los que vive, y en relación con sus objetivos, expectativas, normas y preocupaciones personales. Cuando esta percepción se ve afectada por la presencia de una enfermedad o su tratamiento, se habla de calidad de vida relacionada con la salud $\left(\right.$ CVRS) ${ }^{8}$. Para el presente estudio se adopta el modelo teórico propuesto por el KIDSCREEN Group Europe ${ }^{9}$, que considera la CVRS como una construcción multidimensional que abarca los componentes físicos, emocionales, mentales, sociales y conductuales del bienestar y el funcionamiento según lo perciben los pacientes y / u otras personas.
Existe controversia sobre la CVRS en niños con PC. Por un lado, hay referencia de que es significativamente menor que la de los niños tipo, mientras que otras investigaciones refieren que es similar e incluso reportan mejores resultados en el dominio de entorno escolar ${ }^{10}$. Estas diferencias pueden deberse a variables propias de la condición de discapacidad (severidad del compromiso, patologías asociadas) o externas pero de gran relevancia como son los aspectos económicos socioculturales y familiares ${ }^{11}$.

Tessier et $a l^{10}$ indican que la CV de los niños con PC se deteriora cuando coexisten complicaciones secundarias como dolor, contracturas y deformidades, más que ante la severidad del compromiso o aspectos sociodemográficos. Lai et $a l^{11}$ explican que en los niños con PC severa se evidencian mayores problemas médicos asociados, como son epilepsia y complicaciones respiratorias, sensoriales, cognitivas, del comportamiento, del habla, musculoesqueléticas, entre otras.

Existen diversos factores que influyen en la CVRS de los niños con PC. Radsel et $a l^{12}$, por ejemplo, muestran que la edad más baja es un predictor de mejor CVRS, y lo mismo es reportado por Quiceno y Vinaccia ${ }^{13}$ con población sin patología. Meade y Dowswell ${ }^{14}$ y Surender $e t a l^{15}$, por otro lado, refieren que las niñas con PC tienen tendencia a reportar más baja CV que los niños, pero una mejor percepción respecto al apoyo social y amigos. En otro estudio, Braccialli et $a l^{16}$ hacen mención a que las niñas con PC son más vulnerables durante su tiempo de crecimiento. Asimismo, contrasta y llama la atención cómo Colver et $a{ }^{17}$ encuentran que la CV de los adolescentes con PC no es inferior a sus pares sin discapacidad. Por otra parte, la condición económica es un aspecto que impacta a las familias: según Glinac et $a l^{18}$ los niños y padres de estrato socioeconómico más bajo dan cuenta de una baja CV en términos de puntajes totales, y según Park ${ }^{19}$ existe una pequeña pero creciente evidencia que relaciona discapacidad y pobreza. Finalmente, el ambiente escolar es un soporte positivo en la medida en que, cuando las familias encuentran entornos asequibles que posibilitan el ingreso de los niños a la escuela, se incrementan los puntajes en la calificación de $\mathrm{CV}^{20}$. 
Conocer la CVRS en niños con PC puede favorecer la conciencia sobre la necesidad de desarrollar programas en salud, educación y a nivel comunitario que reduzcan el impacto que genera la PC en los niños y sus familias. Por lo tanto, este trabajo pretende determinar la $\mathrm{CV}$ relacionada con la salud de niños y niñas entre 8 y 12 años con PC y relacionarla con sus características sociodemográficas y clínicas.

\section{MATERIALES Y MÉTODOS}

\section{Tipo de investigación}

Estudio de tipo descriptivo correlacional. Se realizó muestreo no probabilístico en bola de nieve, con el cual se obtuvo muestra de los diferentes estratos socioeconómicos. El tamaño de la muestra se calculó con base en la población total de la ciudad de Cali, Colombia, para 2016, con una frecuencia esperada de 5 niños con PC por cada 1.000 nacidos vivos. El límite de confianza fue de $5 \%$ y la tasa de no respuesta fue de $20 \%$ para un tamaño total de muestra de 100 niños.

\section{Participantes}

La población estuvo conformada por 100 niños con PC, entre 8 y 12 años de edad, con cualquier severidad en su compromiso motor, que asistían al colegio o que realizaban actividades de inclusión identificadas por el niño y la familia como análogas al colegio. No se incluyeron aquellos niños cuyos padres o cuidadores no contaban con tiempo para responder los cuestionarios, niños con enfermedad crónica adicional no relacionada con la PC y familias con otro niño con PC, autismo o discapacidad cognitiva.

Para definir el cálculo de la muestra se tomó como referencia la población total en la ciudad de Cali para 2016, la cual según el Departamento Administrativo Nacional de Estadística (DANE) era de 2.394.870 habitantes. La frecuencia esperada, de $0,5 \%$, se calculó en una prevalencia de 5 niños con PC por cada 1.000 nacidos vivos según lo referenciado para el Perú ${ }^{6}$ ya que en Colombia no se encontraron datos sobre esta condición. El límite de confianza fue de $5 \%$, y la tasa de no respuesta, de $20 \%$.

\section{Instrumentos}

Se usó un cuestionario de caracterización sociodemográfica y clínica elaborado por la investigadora, en donde se consideraron variables como sexo, estrato socioeconómico (según clasificación en seis estratos definida en Colombia por el DANE), grado escolar, tono postural y extensión de la lesión. Asimismo se contempló la Gross Motor Function Classification System (Clasificación de la Función Motora Gruesa - GMFCS-), la cual clasifica la PC en cinco niveles y describe el movimiento realizado por el paciente con énfasis en la sedestación, las transiciones y la movilidad general ${ }^{21}$ así: el niño nivel I camina sin restricciones, los del nivel II caminan con limitaciones, en el nivel III requieren aditamentos de apoyo para la deambulación, en el nivel IV la automovilidad es limitada, y los niños nivel $V$ requieren ser transportados en silla de ruedas.

La CVRS puede ser medida por una variedad de cuestionarios tanto generales como específicos para cada enfermedad $^{22}$. En el presente estudio se usó el cuestionario KIDSCREEN 27 (KS-27) para niños, y en el caso de aquellos niños que no se comunicaban verbalmente, uno de los padres o el cuidador principal respondió el cuestionario KS-27 para padres. El cuestionario KS-27 puede ser usado en niños entre 8 y 18 años $^{23}$, y la versión de 27 ítems para niños y padres ${ }^{24}$ ha sido validada en Colombia con una consistencia interna mayor a 0,7 en todas las dimensiones. En todos los dominios el coeficiente de correlación intraclase fue superior a 0,87 en la fiabilidad interobservador y mayor a 0,8 en la fiabilidad intraobservador ${ }^{25}$. Este instrumento usa una escala de Likert con cinco opciones y solicita la información de lo percibido en la última semana en cinco dimensiones: actividad física y salud, estado de ánimo y sentimientos, vida familiar y tiempo libre, apoyo social y amigos, y entorno escolar y colegio. El puntaje de cada dimensión se calcula dándole un valor de 0 a 4 puntos para cada opción del cuestionario, previa inversión de los ítems negativos, y se lleva a una escala de 0 a 100 en la que los puntajes más bajos reflejan baja calidad de vida ${ }^{26}$. En Colombia se ha usado este cuestionario en pacientes con hemofilia ${ }^{27}$, rinitis y asma alérgica $^{28}$, sobrepeso y obesidad ${ }^{29}$. No hay referencia de su uso o del de otros instrumentos que evalúen la CVRS en niños con PC. 


\section{Declaración sobre aspectos éticos}

Este estudio adoptó las normas establecidas en la Declaración de Helsinki ${ }^{30}$ de 1975 y la Resolución 8430 de 1993 del Ministerio de Salud ${ }^{31}$ para la investigación con seres humanos. A las familias que aceptaron participar, se les entregó y explicó el consentimiento informado, el cual fue firmado por el padre o cuidador principal del niño. El asentimiento informado fue firmado por aquellos niños que funcionalmente podían firmar con su propia mano. Los instrumentos fueron diligenciados por la investigadora principal, y cada documento fue codificado y guardado bajo llave. Se solicitó permiso formal para realizar la investigación a instituciones de salud, educativas, deportivas y recreativas, tanto públicas como privadas, donde habitualmente acuden niños con PC. Se obtuvo carta de aprobación 001-016 del comité institucional de revisión de ética humana de la Universidad del Valle y, para la aplicación de los cuestionarios KS-27 para niños y KS-27 para padres, se contó con el permiso del KS Group.

\section{RESULTADOS}

\section{Análisis estadístico}

En el análisis univariado se reportaron las variables numéricas con las medianas y los valores máximos y mínimos. En el caso de la edad se determinó el promedio y su desviación estándar. Para el análisis bivariado se categorizó la variable edad tanto de los niños como de los cuidadores, y para relacionar las variables con los dominios del KS-27 se usó la prueba chi cuadrado, estableciendo un nivel de significancia del 5\%. Los datos fueron analizados con Stata v.13.

\section{Análisis univariado}

Al explorar los datos se encontró que las variables numéricas no contaron con una distribución normal, excepto la que correspondía a la edad. Estas variables y los datos arrojados por cada uno de los dominios del KS-27 fueron explorados con la prueba de Shapiro-Wilk. De este modo se encontró un valor $p<0,05$, por lo cual se rechazó la hipótesis nula, que indica que los datos se distribuyen normalmente, excepto en el dominio estado emocional y sentimientos (EA). Debido a que esta es la variable respuesta, se reportan las medianas con sus respectivos valores máximos y mínimos.

El $47 \%$ de los cuestionarios aplicados fue respondido por la madre del niño; el $4 \%$, por el padre; el $40 \%$, por el niño con PC, y el 9\%, por otros, entre los que se encontraban abuelos y cuidadores diferentes a la familia. Respecto a las características sociodemográficas de la población, hubo predominio del sexo masculino, con un 53\%. El 51\% de la población total se encontraba entre 10 y 12 años, y el $83 \%$ pertenecía a los estratos 1 a 3 . En cuanto al nivel educativo, el 54\% asistía a la escuela primaria, y el $17 \%$, a programas de inclusión. La PC espástica fue el tipo de tono más frecuente con un $87 \%$, y el $40 \%$ de los niños fueron cuadriparéticos. Según la GMFCS, los niveles II y V representaron, cada uno, el $28 \%$ de la población. Respecto a los cuidadores, el $45 \%$ reportaron secundaria como mayor nivel educativo alcanzado, y el $46 \%$ se encontraba entre 20 y 35 años.

Como se presenta en la tabla 1 , las puntuaciones totales del KS-27 mostraron que el dominio de estado de ánimo y sentimientos fue el mejor calificado, mientras que el de actividad física y salud fue el que obtuvo menor puntaje.

Tabla 1. Dominios de calidad de vida (KIDSCREEN 27).

\begin{tabular}{|c|c|}
\hline Dominios de calidad de vida & Mediana ( mínimo-máximo) \\
\hline Actividad física y salud (AF) & $60(20-100)$ \\
\hline Estado de ánimo y sentimientos (EA) & $82,1(42,8-100)$ \\
\hline Vida familiar y tiempo libre (FL) & $67,8(14,2-100)$ \\
\hline Apoyo social y amigos (AM) & $62,5(0-100)$ \\
\hline Entorno escolar (EC) & $81,2(12,5-100)$ \\
\hline
\end{tabular}




\section{Análisis bivariado}

En la tabla 2 se muestra la distribución de los dominios del KS-27 respecto a las características sociodemográficas de los niños con PC. Se encontró significancia estadística en la relación entre el dominio entorno escolar del KS-27 y la variable grado escolar para todos los grados a excepción de transición, primero y tercero de primaria. Por otra parte, al verificar el comportamiento de los dominios del KS-27 respecto a las variables sociodemográficas, se observó que el dominio de entorno escolar fue el mejor percibido por las niñas, y el de estado de ánimo y sentimientos, por los niños. Los niños más pequeños ( 8 a 9 años) reportaron mejor percepción sobre actividad física y salud que los niños grandes (10 a 12 años), mientras que estos últimos calificaron mejor que los niños menores el dominio de apoyo social y amigos. Los niños de estrato socioeconómico alto $(5$ y 6$)$ describieron las mejores puntuaciones en todos los dominios de CV, a excepción de apoyo social y amigos, el cual fue mejor percibido por los niños de estrato medio; para los niños de estrato bajo la mejor percepción fue para el dominio de entorno escolar. Los niños quienes cursaban bachillerato calificaron con las mejores puntuaciones todos los dominios evaluados, y aquellos que asistían a programas de inclusión manifestaron más baja percepción de actividad física y salud.

Tabla 2. Dominios del KS-27 y características sociodemográficas.

\begin{tabular}{|c|c|c|c|c|c|c|c|}
\hline Var & $\begin{array}{l}\text { ble/dominic } \\
n(\%)\end{array}$ & & $\mathbf{A F}$ & EA & FL & AM & EC \\
\hline \multirow{2}{*}{ Sexo } & Femenino & 47 & $60(20-100)$ & $78,5(42,8-100)$ & $67,8(14,2-100)$ & $75(25-100)$ & $87,5(50-100)$ \\
\hline & Masculino & 53 & $60(20-100)$ & $82,1(50-100)$ & $67,8(28,5-100)$ & $56,2(0-100)$ & $81(12,5-100)$ \\
\hline \multirow{2}{*}{ Edad } & 8 a 9 años & 49 & $71,4(14,2-100)$ & $82,1(42,8-100)$ & $71,(14,2-100)$ & $62,5(0-100)$ & $\begin{array}{c}81,2(12,5- \\
100)\end{array}$ \\
\hline & $\begin{array}{l}10 \text { a } 12 \\
\text { años }\end{array}$ & 51 & $64,2(24,43-100)$ & $82,1(50-100)$ & $64,2(21,43-100)$ & $68,7(0-100)$ & $\begin{array}{c}81,2(37,5- \\
100)\end{array}$ \\
\hline \multirow{3}{*}{$\begin{array}{c}\text { Estrato } \\
\text { socioeco } \\
\text { nómico }\end{array}$} & Bajo:1-2 & 57 & 55 (20-100) & $78,5(42,8-100)$ & $64,2(14,2-100)$ & $56,2(0-100)$ & $\begin{array}{c}81,2(12,5- \\
100)\end{array}$ \\
\hline & Medio: 3-4 & 36 & $62,5(20-90)$ & $82,1(53,5-100)$ & $67,8(21,4-96,4)$ & $78,1(0-100)$ & $\begin{array}{c}81,2(31,2- \\
100)\end{array}$ \\
\hline & Alto: 5-6 & 7 & $85(35-90)$ & $85,7(50-100)$ & $82(46,4-100)$ & $\begin{array}{c}75(37,5- \\
93,7)\end{array}$ & $\begin{array}{c}87,5(62,5- \\
100)\end{array}$ \\
\hline \multirow{2}{*}{$\begin{array}{l}\text { Grado } \\
\text { escolar }\end{array}$} & Talleres & 17 & $45(20-100)$ & $71,4(50-100)$ & $50(28,5-96,4)$ & $50(0-93,7)$ & $\begin{array}{c}68,7(31,2- \\
100)^{*}\end{array}$ \\
\hline & Prejardín & 2 & $30(20-40)$ & $75(42,8-82,1)$ & $60,7(57,1-64,2)$ & $\begin{array}{c}46,8(43,7- \\
50)\end{array}$ & $\begin{array}{c}34,3(12,5- \\
56,2)^{*}\end{array}$ \\
\hline
\end{tabular}


Calidad de vida relacionada con características sociodemográficas y clínicas en niños con parálisis cerebral

\begin{tabular}{|c|c|c|c|c|c|c|}
\hline Jardín & 8 & $40(20-90)$ & $75(42,8-100)$ & $55,3(14,2-89,2)$ & $56,2(0-93,7)$ & $\begin{array}{c}34,3(12,5- \\
56,2)^{*}\end{array}$ \\
\hline Transición & 11 & $45(25-90)$ & $75(50-96,4)$ & $75(28,5-100)$ & $\begin{array}{c}50(12,5- \\
81,2)\end{array}$ & $87,5(50-100)$ \\
\hline Primero & 21 & $70(30-100)$ & $85,7(53,5-100)$ & $67,8(42,8-100)$ & $\begin{array}{c}87,5(25- \\
100)\end{array}$ & $\begin{array}{c}87,5(56,2- \\
100)\end{array}$ \\
\hline Segundo & 13 & $75(20-100)$ & $85,71(60,7-100)$ & $75(28,5-92,8)$ & $\begin{array}{c}75(56,2- \\
100)\end{array}$ & $\begin{array}{c}81,2(56,2- \\
100)^{*}\end{array}$ \\
\hline Tercero & 10 & $57,5(35-90)$ & $78,5(53,5-100)$ & $64,2(21,5-82,1)$ & $\begin{array}{c}78,1(43,7- \\
100)\end{array}$ & $\begin{array}{c}78,1(62,5- \\
100)\end{array}$ \\
\hline Cuarto & 9 & $75(30-85)$ & $89,2(57,1-96,4)$ & $78,5(28,5-96,4)$ & $\begin{array}{c}75(56,2- \\
100)\end{array}$ & $\begin{array}{c}81,2(62,5- \\
100)^{*}\end{array}$ \\
\hline Quinto & 1 & $70(70-70)$ & $71,4(71,4-71,4)$ & $50(50-50)$ & $\begin{array}{c}43,7(43,7- \\
43,7)\end{array}$ & $\begin{array}{c}87,5(87,5- \\
87,5)^{*}\end{array}$ \\
\hline Sexto & 3 & $60(30-80)$ & $85,7(82,1-96,4)$ & $89,2(46,4-82,1)$ & $100(0-100)$ & 75 (75-87.5)* \\
\hline Séptimo & 3 & $50(30-65)$ & $96,4(53,5-100)$ & $82,1(46,4-82,1)$ & $\begin{array}{c}81,2(43,7- \\
93,7)\end{array}$ & $\begin{array}{c}93,7(93,7- \\
100)^{*}\end{array}$ \\
\hline Noveno & 1 & $75(75-75)$ & $100(100-100)$ & $92,8(92,8-92,8)$ & $\begin{array}{c}87,5(87,5- \\
87,5)\end{array}$ & $\begin{array}{c}93,75(93,7- \\
93,7)^{*}\end{array}$ \\
\hline
\end{tabular}

En la tabla 3 se muestra la distribución de los dominios del KS-27 y las variables clínicas. Se encontró que el dominio del KS-27 apoyo social y amigos fue estadísticamente significativo con respecto a espasticidad, hipotonía y ataxia. Las relaciones entre los dominios actividad física y salud, vida familiar y tiempo libre, y apoyo social y amigos con todos los tipos de extensión de la lesión fueron estadísticamente significativas. Asimismo, se halló significancia estadística entre todos los niveles según la GMFCS con el dominio de actividad física y salud. Al verificar el comportamiento de los dominios del KS-27 respecto a las variables clínicas, se observa que los niños con espasticidad señalaron altas puntuaciones en los dominios actividad física y salud, y estado de ánimo y sentimientos. Según la extensión del compromiso motor, los niños con hemiparesia tenían mejor percepción respecto a actividad física y salud, y los niños con diparesia y cuadriparesia percibieron mejor el entorno escolar. Respecto a la funcionalidad, los niños de niveles I y II según la GMFCS describieron las mejores puntuaciones en todos los dominios de CV, y los niños de niveles IV y $\vee$ calificaron mejor el dominio de entorno escolar. 
Tabla 3. Dominios del KS-27 y características clínicas.

\begin{tabular}{|c|c|c|c|c|c|c|c|}
\hline \multicolumn{3}{|c|}{$\frac{\text { Variable/dominio }}{\mathrm{n}(\%)}$} & $\mathbf{A F}$ & EA & FL & AM & EC \\
\hline \multirow{4}{*}{ Tono } & Espasticidad & 87 & $60(20-100)$ & $\begin{array}{c}82,1(42,8- \\
100)\end{array}$ & $\begin{array}{c}67,8(14,2- \\
100\end{array}$ & $\begin{array}{c}62,5(0- \\
100)^{*}\end{array}$ & $\begin{array}{c}81,2(31,2- \\
100)\end{array}$ \\
\hline & Hipotonía & 6 & $55(20-100)$ & $71,4(50-85,7)$ & $\begin{array}{c}62,5(46,4- \\
92,8) \\
\end{array}$ & $\begin{array}{c}56,2(43,7- \\
93,7)^{*} \\
\end{array}$ & $\begin{array}{c}62,5(12,5- \\
81,2) \\
\end{array}$ \\
\hline & Discinesia & 6 & $47,5(35-80)$ & $75(60,7-92,8)$ & $\begin{array}{c}66,0(28,5- \\
92,8)\end{array}$ & $\begin{array}{c}59,3(12,5- \\
93,7)\end{array}$ & $\begin{array}{c}75(68,7- \\
100)\end{array}$ \\
\hline & Ataxia & 1 & $30(30-30)$ & $\begin{array}{c}57,1(56,1- \\
56,1)\end{array}$ & $\begin{array}{c}67,8(67,8- \\
67,8)\end{array}$ & $\begin{array}{c}68,7(68,7- \\
68,7)^{*}\end{array}$ & $\begin{array}{c}87,5(87,5- \\
87,5)\end{array}$ \\
\hline \multirow{3}{*}{ Extensión } & Cuadriparesia & 40 & $40(20-100)^{*}$ & $\begin{array}{c}71,43(42,8- \\
100)\end{array}$ & $\begin{array}{c}53,5(14,2- \\
96,4)^{*}\end{array}$ & $\begin{array}{c}56,2(12,5- \\
100)^{*}\end{array}$ & $\begin{array}{c}75(12,5- \\
100)\end{array}$ \\
\hline & Hemiparesia & 29 & 75 (20-90)* & $\begin{array}{c}85,7(53,5- \\
100)\end{array}$ & $\begin{array}{c}78,5(46,4- \\
96,4)^{*}\end{array}$ & $\begin{array}{l}75(0- \\
100)^{*}\end{array}$ & $\begin{array}{c}81,2(31,2- \\
100)\end{array}$ \\
\hline & Diparesia & 31 & $65(20-100)^{*}$ & $\begin{array}{c}85,7(53,5- \\
100)\end{array}$ & $\begin{array}{c}75(42,8- \\
100)^{*}\end{array}$ & $\begin{array}{c}81,2(0- \\
100)^{*}\end{array}$ & $\begin{array}{c}87,5(37,5- \\
100)\end{array}$ \\
\hline \multirow{5}{*}{ GMFCS } & Nivel I & 21 & 75 (20-90)* & $\begin{array}{c}85,71(50- \\
100)\end{array}$ & $\begin{array}{c}75(46,4- \\
92,8)\end{array}$ & $\begin{array}{c}62,5(0- \\
100)\end{array}$ & $\begin{array}{c}81,2(37,5- \\
100)\end{array}$ \\
\hline & Nivel II & 28 & $\begin{array}{c}72,5(20- \\
100)^{*}\end{array}$ & $\begin{array}{c}86,7(53,5- \\
100)\end{array}$ & $\begin{array}{c}75(46,4- \\
100)\end{array}$ & $\begin{array}{c}81,25(0- \\
100)\end{array}$ & $\begin{array}{c}81,2(31,2- \\
100)\end{array}$ \\
\hline & Nivel III & 10 & $65(30-100)^{*}$ & $\begin{array}{c}89,3(57,1- \\
100)\end{array}$ & $\begin{array}{c}71,4(28,5- \\
96,4)\end{array}$ & $\begin{array}{c}71,8(50- \\
100)\end{array}$ & $\begin{array}{c}87,5(50- \\
100)\end{array}$ \\
\hline & Nivel IV & 13 & $40(30-65)^{*}$ & $\begin{array}{c}78,5(57,1- \\
100)\end{array}$ & $\begin{array}{c}69,7(21,4- \\
89,2)\end{array}$ & $\begin{array}{c}56,2(0- \\
100)\end{array}$ & $\begin{array}{c}87,5(56,2- \\
100)\end{array}$ \\
\hline & Nivel V & 28 & $40(20-80)^{*}$ & $\begin{array}{c}69,6(42,8- \\
100)\end{array}$ & $\begin{array}{c}51,7(14,2- \\
96,4)\end{array}$ & $\begin{array}{c}56,2(12,5- \\
100)\end{array}$ & $\begin{array}{c}75(12,5- \\
100)\end{array}$ \\
\hline
\end{tabular}

${ }^{*}$ Chi cuadrado $\mathrm{p}<0,05$.

En cuanto a la distribución de los dominios del KS-27 respecto a las características sociodemográficas del cuidador (tabla 4), se encontró significancia estadística para el dominio actividad física y salud respecto al sexo, para el dominio de estado de ánimo y sentimientos con relación al nivel educativo de los cuidadores y entre el dominio entorno escolar y el estrato socioeconómico. Al verificar el comportamiento del KS-27 respecto a las variables sociodemográficas del cuidador, se observaron las más altas puntuaciones en todos los dominios, a excepción de vida familiar y tiempo libre, en los niños cuidados por personas del género femenino. Así también, los niños cuidados por personas mayores de 50 años mostraron las más altas calificaciones para todos los dominios del KS-27, a excepción de vida familiar y tiempo libre. En cuanto al nivel educativo, la mejor percepción en todos los dominios del KS-27 se encontró en personas con formación universitaria y posgradual. 
Tabla 4. Dominios del KS-27 y características sociodemográficas del cuidador.

\begin{tabular}{|c|c|c|c|c|c|c|c|}
\hline \multicolumn{3}{|c|}{$\begin{array}{c}\text { Variable/dominio } \\
\mathrm{n}(\%)\end{array}$} & AF & EA & FL & AM & EC \\
\hline \multirow{2}{*}{ Sexo } & Femenino & 96 & $60(20-100) *$ & $\begin{array}{c}82,1(42,8- \\
100)\end{array}$ & $67,8(14,2-100)$ & $62,5(0-100)$ & $\begin{array}{c}81,2(12,5- \\
100)\end{array}$ \\
\hline & Masculino & 4 & $50(45-80)^{*}$ & $\begin{array}{c}78,5(57,1- \\
100)\end{array}$ & $73,2(46,4-85,7)$ & $\begin{array}{c}53,1(43,7- \\
93,7)\end{array}$ & $\begin{array}{c}81,2(62,5- \\
100)\end{array}$ \\
\hline \multirow{3}{*}{$\begin{array}{c}\text { Categorías } \\
\text { de edad }\end{array}$} & 20 a 35 años & 46 & $52,5(20-100)$ & $78,5(50-100)$ & $69,6(21,4-96,4)$ & $\begin{array}{c}62,5(62,5- \\
100)\end{array}$ & $\begin{array}{c}87,5(12,5- \\
100)\end{array}$ \\
\hline & 36 a 50 años & 42 & $60(20-100)$ & $82,1(50-100)$ & $66,0(26,5-100)$ & $62,5(0-100)$ & $\begin{array}{c}81,2(31,2- \\
100)\end{array}$ \\
\hline & $\begin{array}{l}51 \text { y más } \\
\text { años }\end{array}$ & 12 & $65(30-90)$ & $\begin{array}{c}85,2(82,1- \\
89.2) \\
\end{array}$ & $53,5(14,2-96,4)$ & $75(43,7-100)$ & $\begin{array}{c}87,5(62,5- \\
100) \\
\end{array}$ \\
\hline \multirow{3}{*}{$\begin{array}{c}\text { Estrato } \\
\text { socioeconóm } \\
\text { ico }\end{array}$} & Bajo & 56 & $40(20-90)$ & $\begin{array}{c}78,5(53,5- \\
100)\end{array}$ & $60,7(32,1-100)$ & $68,7(0-100)$ & $\begin{array}{c}93,7(56,2- \\
100)^{*}\end{array}$ \\
\hline & Medio & 42 & $30(20-70)$ & $\begin{array}{c}57,1(42,8- \\
89,2)\end{array}$ & $53,5(14,2-67,8)$ & $62,5(25-93,7)$ & $\begin{array}{c}68,7(50- \\
87,5)^{*}\end{array}$ \\
\hline & Alto & 2 & ------------ & |------------ & --------------- & --------------- & ------------- \\
\hline \multirow{5}{*}{$\begin{array}{c}\text { Nivel } \\
\text { educativo }\end{array}$} & Primaria & 22 & $57,5(25-90)$ & $\begin{array}{c}85,7(42,8- \\
96,4)^{*}\end{array}$ & $64,2(14,2-96,4)$ & $59,3(0-100)$ & $\begin{array}{c}87,5(37,5- \\
100)\end{array}$ \\
\hline & Secundaria & 45 & $60(20-100)$ & $\begin{array}{c}78,5(53,5- \\
100)^{*}\end{array}$ & $67,8(28,5-100)$ & $68,7(25-100)$ & $81,2(50-100)$ \\
\hline & Tecnología & 13 & $45(20-90)$ & $75(53,5-100)^{*}$ & $53,5(21,4-82,1)$ & $50(0-100)$ & $\begin{array}{c}81,2(12.5- \\
100)\end{array}$ \\
\hline & Universitario & 17 & $70(30-100)$ & $85,7(50-100) *$ & $\begin{array}{c}85,7(21,43- \\
82,14)\end{array}$ & $81,2(0-100)$ & $75(50-100)$ \\
\hline & Posgrado & 3 & 75 (35-85) & $\begin{array}{c}60,7(50- \\
82,1)^{*}\end{array}$ & $\begin{array}{c}64,29(46,4- \\
96,4)\end{array}$ & $\begin{array}{c}93,7(62,5- \\
100)\end{array}$ & $\begin{array}{c}68,7(62,5- \\
81,2)\end{array}$ \\
\hline
\end{tabular}

${ }^{*}$ Chi cuadrado $\mathrm{p}<0,05$.

\section{DISCUSIÓN}

La presente investigación es de utilidad para los profesionales de la salud y del área educativa, así como para los entes gubernamentales, pues permite dimensionar la importancia de implementar programas de educación a la comunidad y de apoyo socioeconómico e inclusión escolar para las familias de niños con PC, lo cual puede redundar en mejorar su CV.

En este estudio el mayor porcentaje de cuidadores fue de sexo femenino. En esa misma línea, Braccialli et $a l^{16}$ encontraron que mayoritariamente los cuidadores de los niños con PC son las madres y tienen un nivel educativo más bajo que el referido por los padres. En cuanto al nivel educativo de los cuidadores, se encontró que quienes contaban con educación universitaria o posgradual refirieron mejor CVRS de los niños en el dominio de actividad física y salud, lo cual es similar a lo reportado por Badia et $a l^{32}$ iError! Marcador no definido., quienes en su investigación con niños con enfermedades crónicas observaron una tendencia a referir mejor bienestar en el funcionamiento físico de los niños de padres con más alto nivel educativo. Estos resultados podrían sugerir que los padres que han logrado una formación escolar más alta cuentan con criterio para buscar alternativas de intervención en rehabilitación más acordes a las necesidades de sus niños. Sin embargo, llama la atención lo reportado por Berman et $a l^{33}$ con niños tipo en los que se usó el KS-27 y en quienes encontraron una relación inversa entre mayor grado educativo de los padres y el bienestar de los niños.

Los niños más comprometidos físicamente, niveles IV y V según la CFMG, percibieron más baja CVRS. Lai 
et $a l^{11}$ y Duvignau-Dondé et $a l^{34}$ reportaron que los niños de niveles IV y $\mathrm{V}$ presentan un mayor decremento en su CV con respecto a los niños menos comprometidos. Park ${ }^{19}$ también refiere que la gravedad de la PC está asociada con una CV física baja. En definitiva, los niños severamente comprometidos a nivel físico tienen mayor dependencia para la realización de las actividades de la vida cotidiana; esta realidad tiene implicaciones sociales pues las personas con discapacidad tienen los mismos derechos que los demás ciudadanos, como se indica en la teoría de derechos humanos ${ }^{35}$. Así, la accesibilidad arquitectónica y el transporte accesible son consideraciones prioritarias que aún requieren de intervención a nivel gubernamental. Por otro lado, los profesionales de la salud, y específicamente de la rehabilitación, al ordenar y entrenar en el uso de ayudas para el desplazamiento y la comunicación en población severamente comprometida, posibilitan mayores oportunidades para la inclusión, la participación y el bienestar de los niños con PC.

En la calificación global de los dominios del KS-27, se encontró que la dimensión de vida familiar y tiempo libre obtuvo un puntaje más bajo que entorno escolar y estado de ánimo y sentimientos. Esto se debe a que las familias de los niños con PC viven una dinámica compleja por la asistencia frecuente a consultas médicas y de rehabilitación, la incertidumbre por el proceso de desarrollo de los niños y la evidente restricción del acceso a diferentes lugares de recreación y al uso del tiempo libre. Otro aspecto para tener en cuenta es el hecho de que si el niño con PC tiene alteraciones cognitivas o de comportamiento, como lo mencionan Pousada et $a l^{36}$, se genera mayor estrés y depresión y la participación en actividades con pares, familia y otros se ve más limitada. Debería ser entonces un compromiso del equipo de profesionales de la salud, y especialmente de los psicólogos y trabajadores sociales, fortalecer a los padres y a las familias en sus competencias para enfrentar la vivencia con un niño con discapacidad múltiple.

Otra dimensión sobre la que se interroga en el cuestionario KS-27 es el apoyo social y amigos. En la presente investigación, de manera general, esta fue la dimensión con más baja calificación después de actividad física y salud. Badia et a ${ }^{32}$ mencionan que la CVRS es mejor cuando existe una red de apoyo que facilite la participación de los niños en actividades de disfrute y ocio, lo cual va a influir positivamente en todos los aspectos de la vida. Podría pensarse que los niños con PC están más solos y tienen menos relación con sus pares, lo que puede deberse por ejemplo a barreras sociales y de accesibilidad. Así pues, todos los profesionales de la salud, del sector educativo y de la comunidad en general pueden comprometerse a que los niños con PC tengan mejores posibilidades de inclusión y participación en los diversos contextos.

En este estudio el entorno escolar fue uno de los mejores calificados, lo que hace suponer que asistir al colegio desempeña un papel importante en mejorar la CV del niño con PC. En este aspecto es clave el enfoque de educación inclusiva. La Organización de las Naciones Unidas para la Educación, la Ciencia y la Cultura (Unesco) ${ }^{37}$ define la inclusión como el proceso que permite tener en cuenta la diversidad de necesidades de todos los estudiantes a través de una mayor participación en el aprendizaje, las actividades culturales y comunitarias. En ocasiones, para el caso de los niños con PC se requiere de ajustes a la estructura física de las instituciones educativas y de adecuaciones organizativas en el aula, en el currículo y en las prácticas pedagógicas de los maestros con el propósito de lograr que el niño participe en los procesos de aprendizaje, disminuya la dependencia del adulto y desarrolle competencias acordes a lo esperado en sus pares, teniendo en consideración la severidad de su compromiso. Así, el enfoque de educación inclusiva brinda oportunidades para que los niños con PC puedan participar, ser incluidos y aceptados. Como lo mencionan Braccialli et $a l^{16}$, el colegio facilita la participación en un ambiente que enriquece de experiencias, permite mejorías en el funcionamiento y en la actividad física e incrementa la sensación de bienestar emocional.

Una limitación de este estudio se debió a que un alto porcentaje de la población de niños con PC no lograba comunicarse, por lo que en esos casos el cuestionario KS-27 fue respondido por los padres y otros cuidadores. Otra limitación importante fue la heterogeneidad y el tamaño de la muestra, lo cual pudo ser la razón por la que se afectó la significancia estadística al estimar la relación entre los dominios de CVRS y las variables independientes, de tal 
manera que no se puede hacer generalización de los hallazgos.

Futuras investigaciones podrían establecer una línea de base de la CV de los niños tipo y contrastarla con la de niños con PC, al igual que comparar la percepción que tienen sobre la CVRS una población de padres y la de sus hijos con PC.

\section{CONCLUSIÓN}

El más alto nivel educativo de los cuidadores, el mayor estrato socioeconómico, una menor severidad en el compromiso motor y estar incluidos en el entorno escolar se relacionan con una mejor CVRS en los niños con PC.

\section{DECLARACIÓN SOBRE CONFLICTOS DE INTERESES}

La autora declara no tener ningún conflicto de interés.

\section{REFERENCIAS BIBLIOGRÁFICAS}

1. Hurtado IL. La parálisis cerebral. Actualización del concepto, diagnóstico y tratamiento. Pediatría integral [revista en la internet]. 2007 Nov [citado 19 abr 2019]; (8): 687-690. Disponible en:

http://www.fundacionobligado.org.ar/wpcontent/uploads/2012/08/Actualizacion-delConcepto.pdf.

2. Rosenbaum $P$, Paneth $N$, Leviton $A$, Goldstein $M$, Bax $M$, Damiano $D$, et al A report: the definition and classification of cerebral palsy April 2006. Dev Med Child Neurol Suppl. 2007; 109: 8-14. https://doi.org/10.1111/j.1469-

8749.2007.tb12610.x.

3. Castellanos GR, Rodríguez SR, Castellanos MR. Definición y clasificación de la parálisis cerebral: ¿un problema ya resuelto? Revista de Neurología. 2007; 45(2): 110-117. https://doi.org/10.33588/rn.4502.2006595.

4. Burton A. Fighting cerebral palsy in Africa. The Lancet Neurology. 2015; 14(9): 876-877. https://doi.org/10.1016/S1474-4422(15)00189-

1.
5. Taboada-Lugo, N., Quintero-Escobar, K., Casamajor-Castillo, M., González-Torres, K., Marrero-Infante, et al Epidemiología de la parálisis cerebral en el Estado Plurinacional de Bolivia, 2009-2012. Revista Peruana de Epidemiología [revista en la internet]. 2013 [citado 19 abr 2019]; 17(2): 1-7. Disponible en: http://www.redalyc.org/articulo.oa?id=203129 458006.

6. Vila JR, Espinoza IO, Guillén D, Samalvides F. Características de pacientes con parálisis cerebral atendidos en consulta externa de neuropediatría en un hospital peruano. Revista Peruana de Medicina Experimental y Salud Pública. 2016; 33: 719-724. https://doi.org/10.17843/rpmesp.2016.334.25 57.

7. Colombia. Ministerio de Salud y de Protección Social. Análisis descriptivo de indicadores. Dirección de epidemiología y demografía. 2014 [Internet]. Bogotá: OND [citado abr 2019]. Disponible en: https://discapacidadcolombia.com/phocadown loadpap/ESTADISTICAS/L\%C3\%ADnea\%20Base \%20Discapacidad\%200ND.pdf.

8. Dobhal, M. Juneja, M. Jain, R. Sairam, S. Thiagarajan, D. Health-related Quality of Life in Children with Cerebral Palsy and Their Families. Indian Pediatrics. 2014; 51: 385- 387. https://doi.org/10.1007\%2Fs13312-014-0414z.

9. Gaspar T, Matos M. Qualidade de vida em crianças e adolescentes versão portuguesa dos intrumentos Kidscreen, 52. Lisboa: FCT; 2008; 30- 31 [citado 25 jun 2019]. Disponible en: https://www.researchgate.net/publication/235 929490.

10. Tessier D, Hefner J, Newmeyer A. Factors related to psychosocial quality of life for children with cerebral palsy. International journal of pediatrics. 2014; 2014. http://dx.doi.org/10.1155/2014/204386.

11. Lai C-J, Chen C-Y, Chen C-L, Chan P-YS, Shen IH, Wu C-Y. Longitudinal changes in health-related quality of life in preschool children with cerebral palsy of different levels of motor severity. Research in Developmental Disabilities. 2017; 
61:

11-18.

https://doi.org/10.1016/j.ridd.2016.11.013.

12. Radsel A, Osredkar D, Neubauer D. Healthrelated quality of life in children and adolescents with cerebral palsy. Slovenian Journal of Public Health. 2017; 56(1): 1-10. https://doi.org/10.1515/sjph-2017-0001.

13. Quiceno JM, Vinaccia S. Calidad de vida relacionada con la salud infantil: una aproximación desde la enfermedad crónica. Psychologia [revista en la internet]. 2013 [citado $19 \mathrm{abr}$ 2019]; 7(2): 69-86. Disponible en: https://www.redalyc.org/pdf/2972/297229855 006.pdf.

14. Meade T, Dowswell E. Adolescents' healthrelated quality of life (HRQoL) changes over time: a three year longitudinal study. Health and quality of life outcomes. 2016; 14(1): 14. https://doi.org/10.1186/s12955-016-0415-9.

15. Surender S, Gowda VK, Sanjay K, Basavaraja G, Benakappa N, Benakappa A. Caregiver-reported health-related quality of life of children with cerebral palsy and their families and its association with gross motor function: A South Indian study. Journal of neurosciences in rural practice. 2016; 7(2): 223-227. 10.4103/09763147.178657.

16. Braccialli LMP, Silva MZ, Braccialli AC, Sankako AN, Araújo R. Impact of school participation on quality of life of Brazilian children with cerebral palsy. International Journal on Disability and Human Development. 2016; 15(1): 23-27. https://doi.org/10.1515/ijdhd-2014-0024.

17. Colver A, Rapp M, Eisemann N, Ehlinger V, Thyen $\mathrm{U}$, Dickinson HO, et al Self-reported quality of life of adolescents with cerebral palsy: a crosssectional and longitudinal analysis. The Lancet. 2015; 385(9969): 705-716. https://doi.org/10.1016/S0140-6736(14)612290 .

18. Glinac A, Tahirović $H$, Delalić A. Family socioeconomic status and healthrelated quality of life in children with cerebral palsy: assessing differences between clinical and healthy samples. Paediatrics Today. 2013; 9(2): 183-191. https://doi.org/10.5457/p2005-114.74.
19. Park E-Y. Relationship between activity limitation and health-related quality of life in school-aged children with cerebral palsy: a cross-sectional study. Health and quality of life outcomes. $\quad 2017 ; \quad 15(1)$ : 87. https://doi.org/10.1186/s12955-017-0650-8.

20. Furtado SR, Sampaio RF, Kirkwood RN, Vaz DV, Mancini MC. Moderating effect of the environment in the relationship between mobility and school participation in children and adolescents with cerebral palsy. Brazilian journal of physical therapy. 2015(AHEAD): 311$319 . \quad$ http://dx.doi.org/10.1590/bjptrbf.2014.0127.

21. Palisano $R$, Rosenbaum $P$, Bartlett $D$, Livingston $M$. Content validity of the expanded and revised Gross Motor Function Classification System. Dev Med Child Neurol. 2008; 50: 744-750. http://dx.doi.org/10.1111/j.14698749.2008.03089.

22. Delor RER, Medina ICC. Calidad de vida en adultos del Paraguay con enfermedad celiaca. Duazary. 2018; 15(1): 61-70. https://doi.org/10.21676/2389783X.2026.

23. Ravens-Sieberer $U$, Gosch $A$, Abel T, Auquier $P$, Bellach B-M, Bruil J, et al Quality of life in children and adolescents: a European public health perspective. Sozial-und Präventivmedizin. 2001; 46(5): 294-302. https://doi.org/10.1007/BF01321080.

24. Vélez CM, Lugo LH, García HI. Validez y confiabilidad del 'Cuestionario de calidad de vida KIDSCREEN-27'versión padres, en Medellín, Colombia. Revista Colombiana de Psiquiatría. 2012; 41(3): 588-605. https://doi.org/10.1016/S0034-7450(14)600317.

25. Quintero CA, Lugo LH, García HI, Sánchez A. Validación del cuestionario KIDSCREEN-27 de calidad de vida relacionada con la salud en niños y adolescentes de Medellín, Colombia. Revista colombiana de Psiquiatría. 2011; 40(3): 470$487 . \quad$ https://doi.org/10.1016/S00347450(14)60141-4.

26. Higuita-Gutiérrez LF, Cardona-Arias JA. Calidad de vida de adolescentes escolarizados de 
Medellín-Colombia, 2014. Revista Facultad Nacional de Salud Pública. 2016; 34(2): 145-155. DOI: 10.17533/udea.rfnsp.v34n2a03.

27. Castaño AF, Restrepo MJ, Durán FS. Calidad de vida en una población con hemofilia: estudio de corte transversal en un centro de tratamiento de hemofilia. Revista Colombiana de Reumatología. 2017; 24(1): 18-24. https://doi.org/10.1016/j.rcreu.2016.10.006.

28. Yepes-Núñez JJ, Gómez C, Espinoza Y, Cardona R. Impacto de la inmunoterapia subcutánea con Dermatophagoides farinae y Dermatophagoides pteronyssinus sobre la calidad de vida de pacientes con rinitis y asma alérgica. Biomédica. 2014; $34(2)$. http://dx.doi.org/10.7705/biomedica.v34i2.174 4.

29. Rodríguez Barrera JC, Bastidas M, Giuseppe G, Olaya-Contreras P. Calidad de vida relacionada con la salud en escolares de 10 a 14 años con sobrepeso y obesidad en la ciudad de Medellín, Colombia. Universitas Psychologica. 2016; 15(2): $\quad 301-314$. http://dx.doi.org/10.11144/Javeriana.upsy152.cves.

30. Asociación Médica Mundial. Declaración de Helsinki. Principios éticos para la investigación médica con sujetos humanos Adoptada por la XVII Asamblea Mundial de la Asociación Médica Mundial Helsinki, Finlandia; 1964. http://dx.doi.org/10.4067/S1726569X2000000200010.

31. Colombia. Ministerio de Salud. Resolución 008430 de 1993, por la cual se establecen las normas científicas, técnicas y administrativas para la investigación en salud [internet]. Bogotá: El Ministerio; 1993 [citado 29 jul 2019]. Disponible en: https://www.minsalud.gov.co/sites/rid/Lists/Bi bliotecaDigital/RIDE/DE/DIJ/RESOLUCION8430-DE-1993.PDF.

32. Badia $M$, Orgaz MB, Gómez-Vela $M$, Verdugo $M A$, Ullan AM, Longo E. Do environmental barriers affect the parent-reported quality of life of children and adolescents with cerebral palsy? Research in developmental disabilities. 2016;
49:

312-321. https://doi.org/10.1016/j.ridd.2015.12.011.

33. Berman AH, Liu B, Ullman S, Jadbäck I, Engström $K$. Children's quality of life based on the KIDSCREEN-27: child self-report, parent ratings and child-parent agreement in a Swedish random population sample. PloS one. 2016; 11(3): e0150545. https://doi.org/10.1371/journal.pone.0150545.

34. Duvignau-Dondé E, Santiago-Vargas JG, OvalleLeyva M, López-Loredo PE, Mejía-Barona M. Calidad de vida en pacientes pediátricos y adolescentes con parálisis cerebral. Revista de Sanidad Militar [revista en la internet]. 2015 [citado 19 abr 2019]; 69(6): 535-542. Disponible en: https://www.medigraphic.com/cgi$\mathrm{bin} / \mathrm{new} /$ resumen.cgi?IDARTICULO=62579.

35. Cuenca Gómez P. Sobre la inclusión de la discapacidad en la teoría de los derechos humanos. Revista de Estudios Políticos. 2012; 158: 103-137 https://doi.org/10.18042/39941.

36. Pousada M, Guillamón N, Hernández-Encuentra $E$, Muñoz E, Redolar D, Boixadós $M$, et al Impact of caring for a child with cerebral palsy on the quality of life of parents: a systematic review of the literature. Journal of Developmental and Physical Disabilities. 2013; 25(5): 545-577. https://doi.org/10.1007/s10882-013-9332-6.

37. Organización de las Naciones Unidas para la Educación, la Ciencia y la Cultura. Superar la exclusión mediante planteamientos integradores en la educación: un desafío y una visión, documento conceptual [internet]. París: Unesco; 2003 [citado 29 jul 2019]. Disponible en:

https://unesdoc.unesco.org/ark:/48223/pf0000 134785_spa. 